\title{
EFEITO DE TERCEIRA PESSOA E DISTÂNCIA SOCIAL: PERCEPÇÃO DA INFLUÊNCIA DOS MEDIA NO EU, NO SEMELHANTE E NO OUTRO NA PROPAGANDA DA PREFEITURA MUNICIPAL DE SALVADOR
}

\author{
THE THIRD PERSON EFFECT \\ AND SOCIAL DISTANCE: PERCEIVED INFLUENCE OF THE MEDIA \\ ON THE SELF, THE SIMILAR AND THE OTHER IN THE ADVERTISING OF \\ THE SALVADOR CITY HALL
}

\begin{abstract}
EFECTO DE TERCERA PERSONA Y DISTANCIA SOCIAL: PERCEPCIÓN DE LA INFLUENCIA DE LOS MEDIA EN SÍ MISMO, EN EL SIMILAR Y EN EL OTRO EN LA PUBLICIDAD DE LA MUNICIPALIDAD PREFECTURA DE SALVADOR
\end{abstract}

\begin{abstract}
Maria Paula Almada e Silva Doutoranda em Comunicação e Culturas contemporâneas (UFBA) mariapaula.almada@gmail.com

Graça Penha Nascimento Rossetto Doutoranda em Comunicação e Culturas contemporâneas (UFBA) gracapnr@gmail.com
\end{abstract}

\begin{abstract}
Resumo
$\mathrm{O}$ efeito de terceira pessoa é um fenômeno sobre o qual se observa uma tendência individual de se superestimar a influência de uma mensagem sobre os outros e de subestimá-la em si. No seu desdobramento avalia-se diversas variáveis, entre elas a hipótese da distância social, segundo a qual os indivíduos tendem a acreditar que os media exercem influência maior em desconhecidos do que naqueles que fazem parte do seu ciclo social. Neste contexto, o objetivo deste artigo é testar a hipótese do efeito de terceira pessoa a partir de uma survey a respeito da publicidade realizada pela prefeitura de Salvador sobre a taxa de desemprego municipal observando sua influência na opinião pública presumida. Conclui-se que tanto a hipótese inicial do efeito quanto a da distância social se confirmam, sendo a percepção do impacto dos media na formação da opinião dos outros maior que na formação da opinião daqueles pertencentes ao ciclo social do indivíduo.
\end{abstract}

Palavras-chave: Efeito de terceira pessoa. Distância social. Influência dos media.

\section{Abstract}

The third person effect is a phenomenon on which there is an individual tendency to overestimate the influence of a message on others and underestimate it on the self. In its unfolding it is possible to assess several variables, including the hypothesis of social distance, whereby individuals tend to believe that the media exert greater influence 
on strangers rather than on those that are part of his social circle. In this context, the aim of this paper is to test the third person effect hypothesis from a survey about the advertising made by the Salvador City Hall about the local unemployment rate, observing its influence on the presumed public opinion. It is concluded that the initial and the social distance hypothesis are confirmed. The perceived impact of the media in shaping the opinions of others was confirmed to be stronger than in shaping the opinions of those that belong to the individual social circle.

Key words: Third person effect. Social distance. Media effects.

\section{Resumen}

El efecto de la tercera persona es un fenómeno en el cual se observa una tendencia individual en sobreestimar la influencia de un mensaje sobre los otros y de subestímala en sí. En su desarrollo avaluase diversas variables, entre ellas la hipótesis de la distancia social, segundo la cual los individuos tienden a creer que los media ejercen una influencia más larga en desconocidos que de los que están en su ciclo social. En ese contexto, el objetivo de ese artículo es testar la hipótesis del efecto de la tercera persona a partir de una investigación acerca de la publicidad echa por la municipalidad de Salvador sobre las tasas de desempleo en la ciudad, observando su influencia en la opinión pública presumida. Se concluye que tanto la hipótesis inicial del efecto cuanto la de distancia social se confirman, siendo la percepción del impacto de los media en la formación de opinión de los otros más larga que en la formación de opinión de los pertenecientes al ciclo social del individuo.

Palabras clave: Efecto en tercera persona. Distancia social. Influencia de los media.

\section{SOBRE O EFEITO DE TERCEIRA PESSOA (ETP)}

A bibliografia a respeito dos efeitos midiáticos permite avaliar que há algumas décadas há um deslocamento do foco dos estudos sobre os efeitos dos meios de comunicação de massa, de uma abordagem a respeito dos efeitos imediatos para os efeitos a longo prazo, influenciando, sobretudo, a construção cognitiva. Isso significa que os efeitos não são manifestados no comportamento explícito da audiência (WOLF, 2001), mas em seu modo de organizar suas representações da realidade que vão além das interações interpessoais vivenciadas.

Neste contexto surge a hipótese do efeito de terceira pessoa (third-person effect), relatada e publicada inicialmente por W. Phillips Davison, em 1983, em um artigo sobre teoria da opinião pública. O pressuposto básico da hipótese é que o indivíduo exposto aos media acredita que uma mensagem comunicativa exerce um efeito mais forte nos outros (terceiros) do que nele mesmo (primeira pessoa). Em linhas gerais, trata-se de um efeito da mensagem sobre a opinião ou crença do indivíduo a respeito dos meios de comunicação. 
No entanto, é preciso ressaltar que o pressuposto de que há um superdimensionamento do efeito sobre os outros supõe que o efeito em questão seja especificamente de uma mensagem, e não dos meios de comunicação de forma genérica.

A hipótese do efeito de terceira pessoa (ETP) é formada por dois componentes, o cognitivo e o comportamental. O primeiro, replicado e comprovado em várias pesquisas (PAUL et al., 2000; PERLOFF, 1993), trata-se de uma mudança que ocorre inicialmente nas crenças dos indivíduos, especificamente sobre a influência das mensagens comunicativas nos outros (terceiros). O segundo está intrinsecamente relacionado ao primeiro, pois à medida que os indivíduos consideram que os efeitos de uma mensagem são mais intensos sobre os outros do que sobre eles mesmos, esboçam uma reação.

Com a passar do tempo e aprimoramento dos estudos que se debruçam sobre a hipótese, os pesquisadores têm buscado compreender de que modo determinadas variáveis influenciam a magnitude do efeito. Dez anos após a publicação do texto inaugural sobre o ETP, Perloff (1993) faz uma revisão crítica e síntese sobre os estudos que derivaram da hipótese de Davison, apontando algumas condições que influenciam a ocorrência do efeito. Exemplos são o teor da mensagem, o nível de envolvimento e conhecimento do indivíduo em relação ao assunto e a credibilidade da fonte. São as chamadas variáveis de influência.

Aqui cabe destacar que o conteúdo da mensagem em questão é geralmente considerado negativo ou socialmente indesejado. Essa é uma característica da mensagem que tem se mostrado essencial para comprovação do efeito, sobretudo para comprovação do seu componente comportamental (GUNTHER e THORSON, 1992). Fatores como o nível de envolvimento (ego-envolvment) e de conhecimento do indivíduo em relação à questão também podem influenciar a intensidade do efeito, visto que, quanto mais o indivíduo sabe sobre determinado tema, ou quanto mais envolvido, menos ele imagina ser persuadido pelos media (PERLOFF, 1989; VALLONE et al., 1985; MUTZ, 1989).

A credibilidade ou a percepção da tendência da fonte também podem aumentar ou diminuir a incidência do efeito. A premissa é que quando o indivíduo percebe que a fonte é negativamente enviesada ou que há intenções persuasivas da parte do comunicador nota-se uma discrepância entre a percepção do efeito da mensagem nos outros e na primeira pessoa (COHEN et al., 1988; GUNTHER, 1991). Há, ainda, a questão da escolaridade que, de acordo com alguns estudos, pode interferir na percepção, de modo que quanto maior o grau de escolaridade do indivíduo, mais significativo é o efeito (GUNTHER, 1995; WILLNAT,1996).

O princípio da percepção de maior efeito é igualmente válido para mensagens persuasivas e também tem sido aplicado à área da publicidade. Os profissionais da 
comunicação, que dominam a gramática dos meios, sabem que aplicando as devidas técnicas são capazes de alcançar uma audiência que pode ser persuadida ou, de forma geral, ter suas opiniões e comportamentos modificados. Assim, (neste caso focando-se na terceira pessoa), utilizam-se de estratégias diversas para persuadir, convencer e influenciar seu público alvo ${ }^{1}$ (BANNING , 2001; CHAPIN, 1999; CHO e BOSTER, 2008; DAY, 2008; DELORME et al., 2006; DELORME et al., 2007; GRIER e BRUMBAUGH, 2007; WHITE e DILLON, 2000).

O ETP também foi verificado nas propagandas políticas, através, por exemplo, da tentativa de convencimento do outro sobre as características positivas de determinado candidato ou, no caso da propaganda negativa, da tentativa de convencimento da incapacidade do oponente ao cargo em disputa (CHRISTEN e GUNTHER, 2003; COHEN e TSFATI, 2009; GUNTHER, 1998; GUNTHER e CHRISTEN, 1999; GUNTHER e CHRISTEN, 2002; MUTZ, 1989; RUCINSKI e SALMON, 1990).

Em virtude disso, e do próprio contexto de surgimento da hipótese, o ETP também se trata de uma abordagem que oferece algum esclarecimento a respeito da formação da opinião pública. Para se aproximar do seu entendimento como "aquelas imagens que são feitas por grupos de pessoas, ou por indivíduos agindo em nome dos grupos” (LIPPMANN, 2008, p. 40), uma questão fundamental é exatamente como as pessoas formam suas crenças sobre o que outras pessoas pensam.

Esse princípio liga-se diretamente à argumentação de Diana Mutz (1989, p. 7), para quem “(...) um resultado influente importante para o estudo da formação da opinião pública é a medida em que as opiniões são expressas publicamente". Nesse mesmo trabalho, para testar e defender a ligação do ETP com formação da opinião pública, ela descreve esse último conceito como mais do que uma coleção de opiniões isoladas e mais do que um mero agregado estatístico. Afirma que "apesar de haver um considerável progresso na nossa habilidade de medir e descrever a opinião pública, para todos os propósitos práticos ela continua um conceito de nível individual” (MUTZ, 1989, p. 7).

$\mathrm{O}$ estudo referenciado se focou nas atitudes em relação à falta de investimentos/interesse financeiro da Universidade de Stanford na África do Sul. Foram feitas uma série de perguntas sobre a opinião dos entrevistados em relação à questão e como eles percebiam ser a opinião pública dentro da comunidade de Stanford. Por fim, para estudar reações de terceira pessoa a percepções da opinião pública, os entrevistados foram

\footnotetext{
${ }^{1}$ Mais detalhes sobre o ETP nas propagandas televisivas ver White e Dillon (2000).
} 
questionados sobre três diferentes aspectos da sua percepção sobre o clima de opinião em torno da questão da falta de investimentos.

Como resultado, chegou-se a conclusão de que as pessoas estimaram que os relatos dos media tiveram maior influência nos outros membros da comunidade de Stanford do que nelas mesmas. Descoberta que, segundo a própria autora, ilustra um aspecto curioso da relação entre comunicação e opinião pública. A comunicação não precisa diretamente afetar opiniões para exercer influência no processo de opinião pública. Nenhuma medida incluída neste estudo envolveu mudança de opinião, mas as implicações para formação da OP se mostraram claras.

Assim, enxerga-se e defende-se que percepções da opinião dos outros, aqui exemplificada pelo ETP, estão inclinadas a desempenhar um papel importante na influência da formação e expressão pública de opiniões.

Não obstante esta ligação, com o passar dos anos e a comprovação dos efeitos cognitivos da abordagem atestada pelas publicações (PAUL et al., 2000; PERLOFF, 1993), os pesquisadores mostravam-se cada vez mais interessados nos possíveis efeitos comportamentais, exemplificados na maioria das vezes pelo apoio à censura. Alguns estudos sobre a pesquisa do efeito de terceira pessoa verificaram que, ao acreditarem que os media exercem grande influência nos outros, os indivíduos, no intuito de proteger os mais influenciáveis pelas mensagens persuasivas, passam a apoiar atitudes censitárias (CHIA, LU e McLEOD, 2004; COHEN e WEIMANN, 2008; McLEOD, EVELAND e NATHANSON, 1997; MEIRICK, 2006; ROJAS, SHAH e FABER, 1996; SALWEN, 1998; SALWEN e DRISCOLL, 1997; SALWEN e DUPAGNE, 1999; YANG, 2005; ZHAO e CAI, 2008).

\subsection{Distância social}

A bibliografia sobre a hipótese do efeito de terceira pessoa revela que para fazer julgamentos precisos a respeito do impacto das mensagens nas pessoas dois tipos de informação são necessárias: (1) a informação a respeito das variáveis e (2) a informação sobre quem são os terceiros em questão. De outra forma, se o conhecimento sobre um ou ambos é indisponível, o exame empírico torna-se deficiente e a probabilidade e dimensão do efeito da mensagem em cada alvo torna-se imprevisível.

Diante disso destaca-se o objeto deste trabalho e a importância de se identificar o outro para observar a ocorrência e magnitude do efeito. Passa-se, então, para uma revisão dos estudos que se preocuparam com a identificação do outro e a influência da distância social na percepção do efeito. O objetivo desta pesquisa é testar se a percepção do efeito é menor 
quando os terceiros são mais próximos do indivíduo questionado (aqueles que fazem parte do seu ciclo social, como parceiro (a), amigo, colega de trabalho ou familiares) do que quando são desconhecidos. Seguindo a mesma lógica, a percepção da influência dos media nas pessoas próximas ao indivíduo deve ainda ser maior que no próprio indivíduo.

Essa discussão data do texto seminal de Davison, questionando se o grau de similaridade entre o indivíduo e o outro é um fator relevante a ponto de influenciar a percepção. A partir daí, alguns pesquisadores passam a se dedicar ao estudo da relação entre o ETP e a proximidade do outro com o indivíduo (ANDSAGER e WHITE, 2007; BRANDT et al., 2009; COHEN et al., 1988; DUCK, 1998; GUNTHER,1991; PEISER e PETER; 2000).

Cohen et al.(1988) são uns dos primeiros a oferecer subsídio empírico à suposição. Avaliando o impacto das notícias entre estudantes da Universidade de Stanford, descobriram que os indivíduos acreditavam que o efeito era maior nos outros estudantes que neles próprios. Ao mesmo tempo, esses indivíduos entendiam que o efeito era ainda maior nos demais californianos do que nos estudantes da Universidade, e mais significativo na opinião pública em geral do que nos outros californianos; comprovando que, quanto mais distante é o outro do eu, mais forte o efeito.

Baseado no princípio da distância social e buscando entender como acontece a percepção das diferenças nas relações interpessoais, Duck (1998) buscou averiguar se há mudança na percepção do ETP quando este é testado através da identidade social (identidade de um grupo) e através da identidade pessoal (identificação entre os indivíduos). Os experimentos mostraram que os fatores de maior influência sobre o efeito de terceira pessoa são as semelhanças e diferenças interpessoais, de modo que, quando há similaridade entre os indivíduos, as percepções de terceira pessoa são moderadas.

Resultados semelhantes foram encontrados por Duck e Mullin (1995) na condução de dois experimentos comparando indivíduos distantes e próximos, para testar se a magnitude da percepção do efeito crescia conforme a distância aumentava. Eles notaram que a percepção da diferença entre o ego e o outro é maior quando se pede que a pessoa julgue o impacto dos media na opinião de um grupo maior, mais distante dele em termos sociais (DUCK e MULLIN, 1995 apud DUCK 1998, p.2).

Em contrapartida, Perloff (1993), Periser e Peter (2000) chamam a atenção para o fato de que esta relação de linearidade entre o efeito e a distância social observada nas investigações de Cohen et al.(1988) e Duck (1998), não foi encontrada no estudo de Cohen e Davis (1991). De acordo com Perloff (1993) as discrepâncias nos resultados relatados pelas pesquisas se deve ao fato de que há diferentes tipos de outros. Por esta razão, ressalta a 
importância de se considerar o grau de proximidade entre os indivíduos nas análises da distância social, o que frequentemente não tem sido feito pelos pesquisadores.

Para aquele autor, a complexidade do conceito se dá por causa da variação entre essa proximidade do outro e do eu, de modo que as pessoas próximas ao indivíduo podem ser muito próximas ou pouco próximas. E isso pode fazer com que o indivíduo perceba, por exemplo, que a influência nos colegas de trabalho é menor que na família, ou vice-versa (PERLOFF, 1993).

\section{OBJETIVO E HIPÓTESES}

Com base nessa literatura, retomamos os principais objetivos que norteiam este artigo para passarmos ao teste empírico. Para isso levantou-se o princípio clássico concernente às pesquisas sobre o ETP, tendo como primeira hipótese que (H1) os indivíduos tendem a acreditar que as mensagens comunicativas exercem influência maior nos outros que neles próprios.

Com o objetivo de constatar a extensão do ETP àqueles que fazem parte do ciclo social dos questionados em primeira pessoa, chega-se à segunda hipótese deste trabalho, sustentando que (H2) os indivíduos tendem a acreditar que as mensagens exercem influência maior em pessoas desconhecidas do que em pessoas que fazem parte do ciclo social do indivíduo; de modo que, quanto maior a distância social entre o indivíduo e o outro, maior a percepção de influência dos media.

Assim, a H2 pretende verificar o efeito da distância social, supondo que o que acontece com a percepção dos indivíduos sobre a influência dos meios de comunicação na formação da opinião segue a seguinte lógica (FIG. 1).:

Figura 1: Ilustração do efeito da distância social. Modelo inspirado no modelo de Andsager e White.

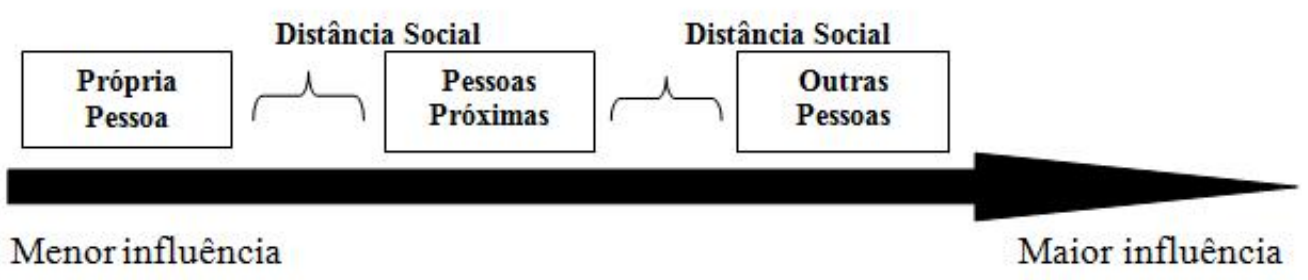

Fonte: ANDSAGER E WHITE, 2007, p. 94. 


\section{ELABORAÇÃO DA PESQUISA}

\subsection{Contexto}

Uma pesquisa recente sobre Emprego e Desemprego (PED), calculada pela SEADE (Fundação Sistema Estadual de Análise de Dados) e pelo DIEESE ${ }^{2}$ (Departamento Intersindical de Estatística e Estudos Socioeconômicos), realizada em sete capitais (Salvador, Distrito Federal, São Paulo, Porto Alegre, Recife, Belo Horizonte e Fortaleza) durante o mês de junho de 2011, aponta Salvador como a capital que apresenta maior índice de desemprego $(15,5 \%)$ quando comparada com as demais (o menor índice foi de 7,7\%, verificado em Belo Horizonte). Por outro lado, o relatório também dá um panorama de como tem evoluído o desemprego de cada uma das cidades avaliadas.

No caso de Salvador, ao mesmo tempo em que a capital apresenta o índice de desemprego mais elevado comparado às demais, este índice, quando relacionado a pesquisas anteriores, levando em conta o desemprego da própria cidade, é o menor desde maio de 1996, quando a pesquisa começou a ser feita. Ainda de acordo com o relatório, o desemprego em Salvador é classificado como estável. No entanto, a prefeitura municipal veiculou, durante o mês de julho, propagandas no rádio e TV comemorando o índice ao ressaltar que Salvador apresenta o menor índice de desemprego desde 1996.

Posta essa situação, observa-se que o relatório pode ser avaliado de duas maneiras, já que ao mesmo tempo em que Salvador teve a pior taxa de desemprego dentre as sete capitais pesquisadas, essa foi também a menor taxa da capital baiana dos últimos anos. Esta situação foi informada aos indivíduos através de um questionário para que eles pudessem avaliar a percepção do efeito de terceira pessoa - gerado pela propaganda da prefeitura municipal neles, nas pessoas próximas a eles, e nos outros de forma geral.

\subsection{Método}

Para testar as hipóteses descritas acima, cento e sete pessoas responderam a um questionário que foi criado no Google Docs e enviado por e-mail para as redes das pesquisadoras. A partir daí o questionário se espalhou, com o método bola de neve, através do pedido das autoras para sua replicação. A única recomendação era que ele fosse enviado somente para moradores da cidade de Salvador, o que está diretamente ligado a questão da importância do envolvimento do entrevistado com a questão. O modo como a situação foi apresentada no questionário baseou-se no primeiro experimento de Davison (1983), que

\footnotetext{
${ }^{2}$ http://www.dieese.org.br/
} 
consiste, em um resumo da situação tratada pelos media e, em seguida, da pergunta sobre se essa notícia influenciaria na formação da opinião dos outros e na formação da opinião do respondente. No caso aqui estudado há ainda um diferencial que merece destaque, que é o esclarecimento ou inclusão de informação a respeito da mensagem midiática veiculada, que adiciona um novo contexto à situação sobre a qual se testa o ETP. Esse trata-se exatamente da explicação sobre a forma como a prefeitura utilizou a informação na sua publicidade, um contexto que poderia ser ou não já observado pelos entrevistados, mas que fica claro ao ser explicitado. Nesse caso o contexto dá um novo entendimento à situação comunicativa, tornando o conteúdo da mensagem indesejável e, assim, colocando-se a situação básica ideal de teste para o ETP.

Dessa forma, o questionário descrevia a situação (explicitada anteriormente) no intuito de inserir o indivíduo naquele contexto e de informá-lo sobre a pesquisa do DIEESE. Após a descrição, o questionário informava que a prefeitura municipal de Salvador tem feito propaganda comemorando a queda do desemprego na capital baiana quando comparado com anos anteriores (como citado acima, é essa a inserção de informação que modifica o contexto). Em seguida, o questionário apresentava três perguntas aos indivíduos: a primeira, se o indivíduo acredita que a propaganda veiculada pela prefeitura pode exercer alguma influência na formação da opinião dos outros sobre a atual gestão municipal. A segunda, se o indivíduo acredita que a propaganda pode exercer alguma influência na formação da sua própria opinião.

Além das duas questões básicas, foi acrescentada a pergunta que pretende verificar a hipótese da distância social. Com esse objetivo, perguntou-se se os indivíduos acreditam que a propaganda veiculada pela prefeitura influenciaria os que são próximos a ele (como amigos, colegas de trabalho, parceiro (a) e familiares).

A mesma escala foi usada para as três perguntas, com as respostas variando de 0 (nenhuma influência) a 4 (total influência). Não foi dada aos respondentes a opção "não tenho opinião a respeito". Cabe pontuar também que foi tomado cuidado para que os indivíduos não suspeitassem quais hipóteses estavam sendo testadas, pois essa descoberta poderia influenciar as respostas.

\subsection{Dados descritivos da amostra}

Dos 107 indivíduos que responderam ao questionário, 42 eram do sexo masculino $(39,3 \%)$ e 65 do sexo feminino (60,7\%). Apresentaram idade entre 18 e 59 anos, fazendo com que a média fosse, aproximadamente, 29 anos. Sobre o nível de escolaridade, 
aproximadamente um terço da amostra $(78,4 \%)$ tem o ensino superior completo; $18,7 \%$ têm o ensino superior incompleto e $6,5 \%$ estão cursando ou já finalizaram o ensino médio.

\section{RESULTADOS}

As tabelas a seguir proporcionam uma visão geral de como se distribuiu o efeito de terceira pessoa de acordo com as respostas obtidas. A primeira tabela mostra como o indivíduo avalia o impacto dos meios de comunicação na formação da sua própria opinião em relação ao impacto dos meios de comunicação na formação da opinião dos outros. Neste caso, a terceira pessoa é alguém distante do respondente, é a população de modo geral.

Com relação à percepção dos indivíduos sobre o impacto dos meios de comunicação na formação de sua própria opinião, $76,7 \%$ acreditam que os meios de comunicação exercem pouca ou nenhuma influência; sendo que 37,4\% delas acreditam que não há qualquer influência. Ainda na formação de suas opiniões, apenas 23,4\% deles acreditam que os meios de comunicação exercem influência significante ou muita influência na formação de suas opiniões ( $15 \%$ e $8,4 \%$ respectivamente).

Quando analisamos o modo como os indivíduos avaliam o impacto dos meios de comunicação na formação da opinião dos outros, os dados praticamente se invertem. Neste caso, podemos observar que os maiores valores encontram-se nas categorias influência significante e muita influência (e não nas categorias nenhuma influência e pouca influência, como observado na análise anterior). Desta maneira, $42,1 \%$ dos indivíduos avaliam que os meios de comunicação exercem influência significante na formação da opinião dos outros, enquanto 33,6\% consideram que os meios de comunicação exercem muita influência na formação da opinião daqueles que elas desconhecem, o que totaliza 75,7\% dos respondentes. Apenas $8,4 \%$ das pessoas foram mais radicais e disseram que os meios de comunicação exercem total influência na formação da opinião dos outros.

Somente $15,9 \%$ dos indivíduos acreditam que os meios de comunicação exercem nenhuma ou pouca influência na formação da opinião dos outros (respectivamente 4,7\% e $11,2 \%$ da amostra). É importante que se observe que estes $4,7 \%$ de indivíduos que disseram que os meios de comunicação exercem nenhuma influência sobre a opinião dos outros também disseram que os meios exercem nenhuma influência sobre si. Porcentagem que, para esta amostra, não demonstra relação significativa. 


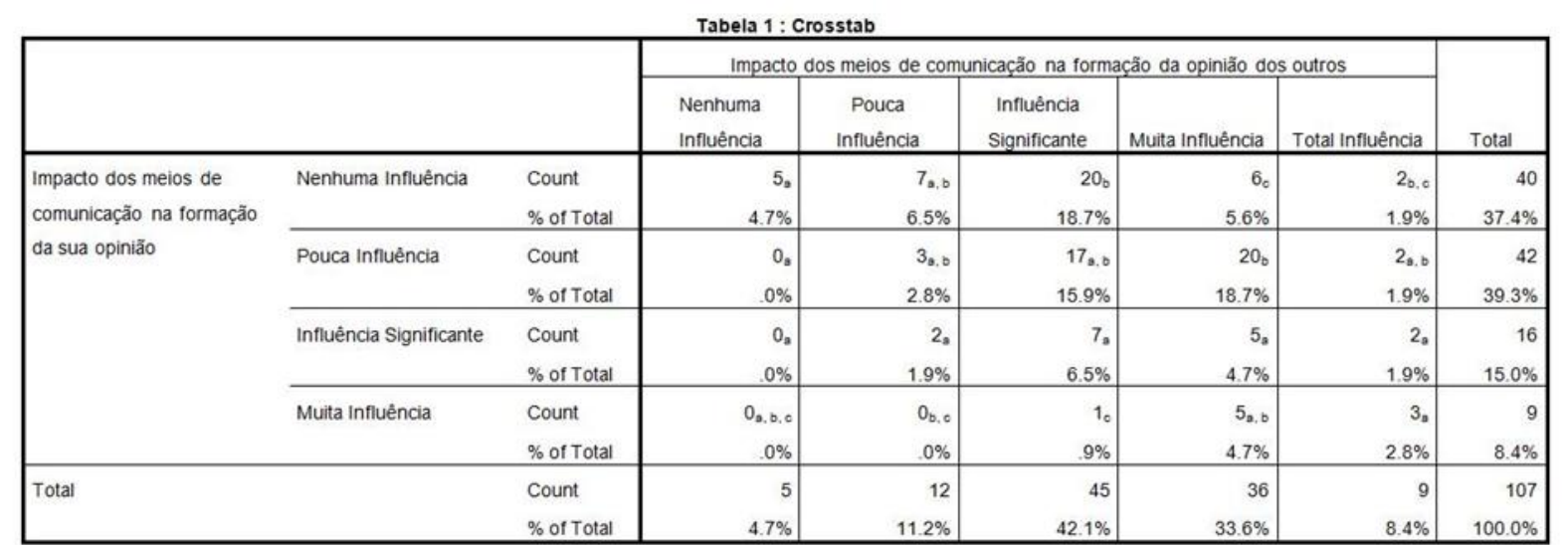

Each subscript letter denotes a subset of Impacto dos meios de comunicação na opinião dos outros categories whose column proportions do not differ significantly from each other at the .05 level.

A segunda tabela mostra como o indivíduo avalia o impacto dos meios de comunicação na formação da sua opinião em relação ao impacto dos meios de comunicação na formação da opinião daqueles pertencentes ao seu ciclo social. Aqui, a terceira pessoa é alguém que o indivíduo conhece e convive e, por essa razão, presume-se que o indivíduo acredita que estas pessoas são menos influenciadas pelos media que os outros a quem desconhece.

O resultado mais significativo da tabela revela que $72 \%$ da amostra acredita que os meios de comunicação exercem pouca ou nenhuma influência na formação da opinião das pessoas próximas a eles; sendo que 54,2\% das pessoas acreditam que há pouca influência e $17,8 \%$ que há nenhuma influência. Ainda, dentre aqueles $54,2 \% ; 29 \%$ responderam que os media também exercem pouca influência sobre a formação da opinião deles próprios. 21,5\% dos respondentes acreditam que os indivíduos do seu ciclo social são influenciados significativamente pelos media, enquanto apenas 6,5\% acreditam que os amigos, parentes, familiares ou parceiros são muito influenciados.

A comparação da última coluna vertical da tabela 1 com a mesma coluna da tabela 2 (chamadas de 'coluna total') mostra que os números que se referem à percepção dos indivíduos sobre o impacto dos meios de comunicação na formação de suas próprias opiniões em ambas as tabelas são os mesmos. Entretanto, como na tabela 2 o impacto do eu é comparado ao impacto das pessoas próximas ao respondente, e na tabela 1 a primeira pessoa é comparada com o impacto dos meios sobre os desconhecidos, a distribuição do efeito dentro da tabela se dá de forma distinta.

Pode-se observar isso, por exemplo, quando se analisa o modo como ocorre a distribuição do efeito entre as 40 pessoas $(37,4 \%)$ que disseram que os meios não exercem nenhuma influência na formação da opinião delas mesmas: na primeira tabela, 20 delas 
responderam que os meios exercem influência significante na formação da opinião dos outros, ao passo que, na segunda tabela, o mesmo número de pessoas (mas não necessariamente as mesmas pessoas) respondeu que os meios exercem pouca influência na formação da opinião das pessoas do mesmo ciclo social.

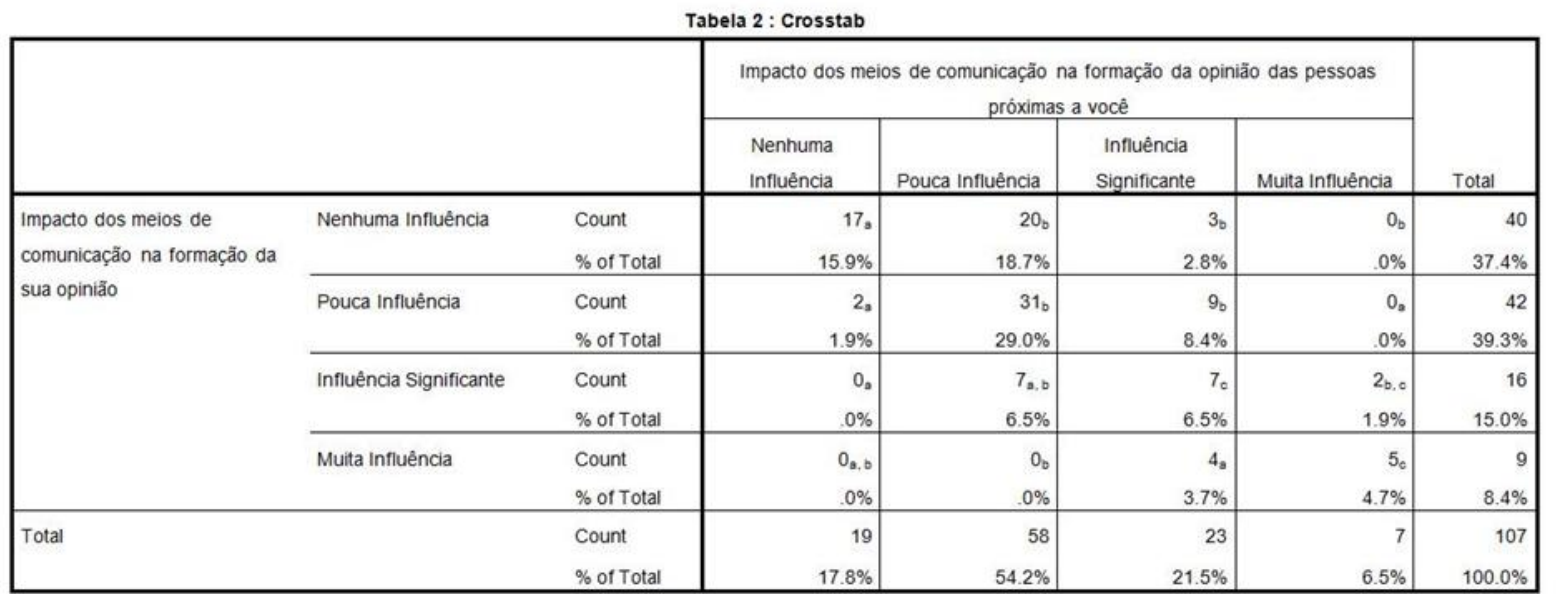

Each subscript letter denotes a subset of Impacto dos meios de comunicação na opinião das pessoas próximas a você categories whose column proportions do not differ significantly from each other at the .05 level.

Tabela 2: relatório de referência cruzada Fonte: Elaboração própria/ SPSS

Tomando como base o grupo de indivíduos que disse que os meios exercem pouca ou nenhuma influência na formação de suas próprias opiniões, vê-se que, quando eles dão suas impressões sobre a influência dos meios na formação da opinião dos outros, $76,82 \%$ das pessoas dizem que os meios exercem significante ou muita influência; e se somarmos a eles os que responderam total influência os números sobem pra $81,70 \%$ dos entrevistados. No entanto, quando a impressão é sobre a formação da opinião de pessoas próximas $85,36 \%$ dos questionados dizem que os meios exercem pouca ou nenhuma influência. A observação destes dados, quando analisados com cautela, sugere indícios de confirmação da hipótese da distância social, pois evidencia a propensão que os indivíduos têm de acreditar que os media exercem influência maior na formação da opinião dos desconhecidos que daqueles que pertencem aos seus ciclos sociais.

Outro indício emerge quando se analisa (1) o total de indivíduos que acredita que os media exercem pouca influência na formação da opinião dos próximos a ele e (2) o total de indivíduos que avalia que os meios de comunicação têm significante ou muita influência na formação da opinião dos outros: 54,2\% dizem que a influência dos media nos amigos, parentes e familiares é pouca; mas $42,1 \%$ e $33,6 \%$ das pessoas dizem que a influência é 
significante e muita, respectivamente (um total de 75,7\%), quando se trata de pessoas que eles não conhecem.

Nota-se, também, que os indivíduos evitam dar respostas extremas. A categoria total influência, por exemplo, não aparece no cruzamento da segunda tabela. Isso significa que nenhum participante acredita que os meios de comunicação exercem total influência sobre eles nem sobre aqueles que lhe são próximos.

\subsection{Testando as hipóteses}

Como se pode ver na tabela 3, ambas as hipóteses se confirmaram. Na segunda coluna são explícitas as médias dos impactos de acordo com as avaliações dos 107 respondentes. Deste modo, pode-se observar que a média do efeito dos meios de comunicação na formação da opinião dos próprios indivíduos é de 0.94. Este resultado é o menor da tabela, o que indica que o próprio indivíduo é aquele que, em sua percepção, sofre menos o efeito dos media.

Tabela 3: Descriptive Statistics

\begin{tabular}{|l|r|r|r|}
\hline & N & Mean & Std. Deviation \\
\hline $\begin{array}{l}\text { Impacto dos meios de comunicaçãona } \\
\text { formação da sua opinião }\end{array}$ & 107 & .94 & .930 \\
Impacto dos meios de comunicaçãona & 107 & 1.17 & .795 \\
formação da opinião das pessoas & & & \\
próximas a você & 107 & 2.30 & .944 \\
$\begin{array}{l}\text { Impacto dos meios de comunicaçãona } \\
\text { formação da opinião dos outros }\end{array}$ & & & \\
& & & \\
Valid N (listwise) & & & \\
\hline
\end{tabular}

Tabela 3: estatística descritiva

Fonte: Elaboração própria/ SPSS

A maior média de impacto (2.30) se refere àquele dos meios de comunicação na formação da opinião dos outros. Isso mostra que, para os indivíduos, os outros - definidos como desconhecidos ou como o público de um modo geral - são os que mais sofrem influência dos meios de comunicação na formação de suas opiniões. Esse resultado confirma a primeira hipótese.

A tabela mostra ainda que os indivíduos veem aqueles próximos a eles como sendo mais influenciados do que eles próprios, mas ao mesmo tempo, menos influenciados que os 
desconhecidos. Isso pode ser afirmado porque a média de influência dos indivíduos próximos à primeira pessoa é 1.17. Esta média se situa entre a média do eu (0.93) e dos outros (2.30), fazendo com que se confirme a segunda hipótese.

\section{DISCUSSÃO}

Tanto a hipótese seminal da pesquisa sobre efeito de terceira pessoa (H1) quanto a hipótese da distância social (H2) foram confirmadas. Entretanto, visto que alguns problemas de ordem metodológica não foram resolvidos, é necessário que algumas ressalvas sejam feitas.

O principal problema de ordem metodológica diz respeito ao fato de que as pessoas próximas aos respondentes foram descritas de modo genérico. Não houve distinção, como sugere Perloff (1993) entre pessoas muito próximas ao indivíduo e pessoas que, apesar de pertencerem ao seu ciclo social, não são tão próximas a ele. No entanto, se reconhece aqui que o ideal seria que, no momento da aplicação do questionário, fosse definido ao indivíduo quem é esse outro pertencente ao seu ciclo social e o quão próxima essa pessoa é dele. A falta dessa diferenciação se deu por uma limitação metodológica na elaboração dos questionários, que, a princípio, não contemplou essa necessidade.

Outro ponto trata-se da avaliação do envolvimento do entrevistado com a questão. Apesar de ser uma variável de influência reconhecida no efeito, neste trabalho ela não pôde ser detalhada. Não foi utilizada nenhuma questão que tornasse possível averiguar quanto o indivíduo conhece ou vive a realidade descrita. Neste caso, portanto, seu envolvimento com a questão limita-se ao fato dele residir na cidade que é objeto do questionário.

Nota-se também que, por vezes, a pesquisa poderia oferecer resultados mais ricos caso fosse acrescentada a variável socioeconômica ao cruzamento das variáveis. Assim, seria possível verificar se a percepção do efeito de terceira pessoa cresce na medida em que cresce o nível socioeconômico dos indivíduos.

Em linhas gerais houve uma certa dificuldade de adaptar o modelo clássico a uma metodologia ideal para medir a percepção do efeito de terceira pessoa. Sabe-se que ela poderia ter sido mais refinada e, ainda, mais abrangente. Contudo, sugere-se que esses esforços sejam superados em futuros trabalhos e reconhece-se que a pesquisa buscou contribuir para um maior aprofundamento dos estudos sobre efeito de terceira pessoa no Brasil.

De toda sorte, cabe destacar que observa-se aqui a pertinência da hipótese em estudo às questões de formação da opinião pública e, consequentemente, de política de forma geral. 
Buscando medir os efeitos imaginados que as mensagens comunicativas podem gerar, a verificação do ETP pode interessar ao campo da comunicação política, sobretudo por entender que como se trata de um efeito presumido a respeito da opinião, a hipótese do dirige nossa atenção às etapas que são formativas da opinião pública. Logo se trata também de formação da opinião pública, sendo essa efeito das mensagens comunicativas em análise.

Essa observação abre um precedente e um extenso campo de estudo a ser explorado pelos pesquisadores da área de comunicação e política, oferecendo a possibilidade de uma rica interação com os estudos de teorias da comunicação e efeitos midiáticos.

\section{REFERÊNCIAS}

ANDSAGER, Julie L.; WHITEe, H. Allen. Self verus others: media, messages and the thirdperson effect. New Jersey: Lawrence Erlbaum Associates, 2007.

BANNING, S. A.: Do You See What I See? Third-Person Effects on Public Communications Through Self-Esteem, Social Stigma, and Product Use. In: Mass Communication \& Society, 2001, 4(2), p. 127-147.

BRANDT, Aafje; VONK, Roos; VAN, Ad Knippenberg. The source effect: Person descriptions by self versus others have differential effects on impression formation.

Personality and Social Psychology Bulletin, 2009, 35(7), p. 965-977.

CHAPIN, John. Third-person perception and sexual risk taking among minority "at-risk" youth. Mass Communication \& Society, 2(3/4), 1999, p. 163-173.

CHIA, S., Lu, K.; McLEOD, D. Sex, Lies, and Video Compact Disc: A Case Study on ThirdPerson Perception and Motivations for Media Censorship. Communication Research, 2004, 31(1), p. 109-130.

CHO, H., BOSTER, F. First and Third Person Perceptions on Anti-Drug Ads Among Adolescents. Communication Research, 2008, 35(2), 169-189.

CHRISTEN, Cindy; GUNTHER, Albert C. The influence of mass media and other culprits on the projection of personal opinion. Communication Research, 2003, 30(4), p. 414-431.

COHEN, J., MUTZ, D., Price, V. and Gunther, A.: Perceived impact of defamation: An experiment on third-person effects. Public Opinion Quarterly, 1988, 52, p. 161 - 73.

COHEN, J., TSFATI, Y. The influence of presumed influence on strategic voting.

Communication Research, 2009, 36(3), p. 359-378.

COHEN, J., WEIMANN, G. Who's Afraid of Reality Shows?: Exploring the Effects of Perceived Influence of Reality Shows and the Concern Over Their Social Effects on Willingness to Censor. Communication Research, 2008, 35(3), p. 382-397. 
DAVISON, W. P. The third-person effect in communication. Public Opinion Quarterly, 1983, 47(1), p. 1-15.

DAY, A. Out of the Living Room and Into the Voting Booth: An Analysis of Corporate Public Affairs Advertising Under the Third-Person Effect. American Behavioral Scientist, 2008, 52(2), p. 243-260.

DELORME, D. E., HUH, J., REID, L. N. Perceived effects of direct-to-consumer (DTC) prescription drug advertising on self and others: A third-person effect study of older consumers. Journal of Advertising, 2006, 35(3), p. 47-65.

DELORME, D. E., HUH, J., REID, L. N. "Other are influenced, but not me": Older adults"e perceptions of DTC prescription drug advertising effects. Journal of Aging Studies, 2007, 21, p. $135-151$.

DUCK, J.; HOGG, M.; THERRY, D. Perceived self-others differences in persuability: the effects of interpersonal and group-based similarity. European Journal of Social Psychology, 1998, 28(1), p. 1-21.

GRIER, S. A., Brumbaugh, A. M. Compared to whom? The impact of status on third person effects in advertising persuasion in a South African context. Journal of Consumer

Behaviour, 2007, 6, p. 5-18.

GUNTHER, A. C. What we think others think: Cause and consequence in the third-person effect, Communication Research, 1991, 18, p. 355-72.

GUNTHER, A., CHRISTEN, C. Effects of news slant and base rate information on perceived public opinion. Journalism and Mass Communication Quarterly, 1999, 76(2), p. 277-292.

GUNTHER, A., CHRISTEN, C. Projection or Persuasive Press? Contrary Effects of Personal Opinion and Perceived News Coverage on Estimates of Public Opinion. Journal of Communication, 2002, 52(1), p. 177-195.

GUNTHER, A., Thorson, E. Perceived persuasive effects of product commercials and public service announcements: Third-person effects in new domains. Communication Research, 1992, 19(5), p. 574-596.

LIPPMANN, W, 1889-1974. Opinião Pública. Petrópolis, RJ: Vozes, 2008.

McLEOD, D., EVELAND, Jr. W. NATHANSON, A. Support for censorship of violent and misogynic rap lyrics: an analysis of the third-person effect. Communication Research, 1997, 24(2), p. 153-174.

MEIRICK, P. Media schemas, perceived effects, and person perceptions. Journalism and Mass Communication Quarterly, 2006, 83(3), p. 632-649.

MUTZ, D. C. The influence of perceptions of media influence: third person effects and the public expression of opinions. International Journal of Public Opinion Research, 1989, $1(1)$, p. 3-23. 
PEISER, W.; PETER, J. Third-person perception of television-viewing behavior. Journal of Communication, 2000, 50 (1), p. 25-45.

PERLOFF, R. Third-person effect research - 1983-1992: A review and synthesis.

International Journal of Public Opinion Research, 1993, 5(2), p. 167-184.

PERLOFF, R. Ego-Involvement and the third person effect of televised news coverage.

Communication Research, 1989, 16(2), p. 236-262.

PRICE, V.; TEWKSBURY, D. Measuring the Third-Person Effect of News: The Impact of Question Order, Contrast And Knowledge. In: International journal of public opinion research, 1996, Vol. 8, No. 2.

ROJAS, H., SHAH, D. FABER, R. For the good of others: censorship and the third-person effect. International Journal of Public Opinion Research, 1996, 8(2), p. 163-186.

RUCINSKI, D., SALMON, C. The 'other' as the vulnerable voter: a study of the third-person effect in the U.S. presidential campaign. International Journal of Public Opinion Research, 1990, 2(4), p. 345-368.

SALWEN, M. B., Perceptions of Media Influence and Support for Censorship: The ThirdPerson Effect in the 1996 Presidential Election. In: Communication Research, 1998, Vol. 25(3), Sage Publications, Inc.

SALWEN, M., DRISCOLL, P. D. Consequences of third-person perception in support of press restrictions in the O. J. Simpson trial. Journal of Communication, 1997, 47(2), p. 6078.

SALWEN, M., DUPAGNE, M. The Third-Person Effect: Perceptions of the Media's Influence and Immoral Consequences. Communication Research, 1999, 26(5), p. 523-549.

VALLONE, R. P., ROSS, L., LEPPER, M. R. The hostile media phenomenon: Biased perception and perceptions of media bias in coverage of the Beirut massacre. Journal of Personality and Social Psychology, 1985, 49(3), p. 577- 585.

WHITE, H. A., DILLON J. F., Knowledge about Other's Reaction to a Public Service Announcement: The Impact on Self Persuasion and Third-Person Perception. In: Journalism as Mass Communication Quarterly, 2000; 77, 4. Pg. 788.

WILLNAT, L. Mass media and political outspokenness in Hong Kong: linking the thirdperson effect and the spiral of silence. International Journal of Public Opinion Research, n. 8,1996, p. $187-211$.

WOLF, M. Teorias da Comunicação. Lisboa: Editorial Presença, 2001.

YANG, K. Consumers' attitudes toward regulation of internet auction sites: A third-person effect perspective. Internet Research, 2005, 15(4), p. 359-367. 
ZHAO, X., CAI, X. From Self-Enhancement to Supporting Censorship: The Third-Person Effect Process in the Case of Internet Pornography. Mass Communication and Society, 2008, 11(4), p. 437-462.

\section{Relatórios:}

Relatório do desemprego das sete capitais pesquisadas pelo DIEESE em junho/2011: http://www.dieese.org.br/ped/peddad.xml (acesso em 20 de julho de 2011)

Relatório do desemprego da Região Metropolitana de Salvador pesquisada pelo DIEESE em junho/2011:

http://www.dieese.org.br/ped/ssa/pedssa0611.pdf (acesso em 20 de julho de 2011).

Original recebido em: 25/08/2013

Aceito para publicação em: 25/06/2014

Sobre os autores

Maria Paula Almada e Silva

Doutoranda e mestre pelo programa de Pós-graduação em

Comunicação e Cultura Contemporâneas da Universidade Federal da Bahia (Pós-com/UFBa) e pesquisadora do Centro de Estudos Avançados em Democracia Digital e Governo Eletrônico (CEADD-UFBA).

Graça Penha Nascimento Rossetto

Doutoranda e mestre pelo programa de Pós-graduação em Comunicação e Cultura Contemporâneas da Universidade Federal da Bahia (Pós-com/UFBa) e pesquisadora do Centro de Estudos Avançados em Democracia Digital e Governo Eletrônico (CEADD-UFBA). 